Ágora Rev. Cient. 2017; 04(02):e7

\title{
La técnica Delphi: Un método de consenso para la investigación en servicios de salud en Latino América
}

\author{
The Delphi technique: A consensus method for research in health services in Latin America \\ Patrick Albert Palmieri ${ }^{1, \mathrm{a}, \mathrm{b}, \mathrm{c}, \mathrm{d}}$
}

RESUMEN

El método de consenso es un grupo de técnicas de investigación utilizado con poca frecuencia para la investigación de servicios de salud en América Latina. El método de consenso proporciona a los investigadores la capacidad de lograr una estimación cuantitativa a través de un enfoque cualitativo. El método de consenso representa un enfoque epistemológico de las ciencias inexactas. Este método que incluye el grupo nominal y la técnica Delphi es útil cuando se requiere un acuerdo de expertos sobre la formulación de recomendaciones, especialmente cuando la evidencia científica no está disponible, las evidencia existentes se debate, o si existe un pequeño equilibrio entre el riesgo-beneficio. El objetivo general es llegar a una conclusión válida, o consenso, de una deliberación grupal repetitiva en respuesta a una pregunta de investigación bien definida. La técnica Delphi es un método esencial para lograr consenso en áreas donde no existía anteriormente evidencia, es limitada, de baja calidad y / o sujeta a debate. Existen cuatro áreas generales en las que los investigadores deberían considerar la técnica Delphi, que incluyen 1) El problema de la investigación no se presta a técnicas analíticas precisas, pero puede beneficiarse de juicios subjetivos sobre una base colectiva; 2) La población de investigación presenta diversos antecedentes con respecto a la experiencia y pericia; 3) Se requieren más sujetos que puedan interactuar eficazmente frente a frente; y 4) El tiempo, los costos y la logística harían inviable las reuniones frecuentes de todos los involucrados. La técnica Delphi crea un entorno estructurado para que los expertos evalúen la mejor información disponible relacionada a una pregunta de investigación y formulen soluciones para responder a esta pregunta a través de un proceso concreto. En general, la técnica Delphi es un poderoso método de investigación cualitativo que debe utilizarse en la investigación de servicios de salud en América Latina.

Palabras clave: consenso, Delphi, cualitativo, resolución de problemas, panel de expertos, análisis crítico

\begin{abstract}
The consensus method is an infrequently utilized group of research techniques for health services research in Latin America. The consensus method derives provides researchers with the ability to achieve a quantitative estimate through a qualitative approach. The consensus method represents an epistemological approach to the inexact sciences. The consensus method, including the nominal group and Delphi techniques, are useful when expert agreement on the formulation of recommendations is required, especially when scientific evidence is unavailable, existing evidence is disputed, or there is a little risk-benefit balance. The overall goal is to arrive at a valid conclusion, or consensus, from a repetitive group deliberation in response to a well-defined research question. The Delphi technique is an essential method to achieve consensus in areas where none previously existed, or the evidence is limited, low quality, and/or subject to debate. There are four general areas when researchers should consider the Delphi technique, including 1) The research problem does not lend itself to precise analytical techniques but can benefit from subjective judgments on a collective basis; 2) The research population presents diverse backgrounds with respect to experience and expertise; 3 ) More subjects are required than can effectively interact face-to-face; and 4) Time, costs, and logistics would make frequent meetings of all subjects unfeasible. The Delphi technique creates a structured environment for experts to review the best available information specific to a research question to formulate solutions to answer the question through a robust process. Overall, the Delphi technique is a powerful qualitative research method that needs to be utilized in Latin American health services research.
\end{abstract}

Key words: consensus, Delphi, qualitative, problem-solving, expert panel, critical analysis

${ }^{1}$ DHSc, EdS, MBA, MSN, PGDip(Oxon), ACNP, RN, CPHRM, CPHQ, FACHE, FISQua, FAAN.

${ }^{a}$ Margaret Jean Watson Profesor Emblemático. Escuela de Enfermería. Universidad Norbert Wiener

${ }^{b}$ Profesor Adjunto. Programa de Doctorado de Ciencias de la Salud. A. T. Still University.

'Profesor Adjunto. Programa de Doctorado en Enfermería. Walden University

'Profesor Adjunto. Nelda C. Stark Escuela de Enfermería. Texas Woman's University. 


\section{INTRODUCTION}

Un importante pero poco reconocido grupo de técnicas de investigación para los de servicios de salud en Latino América, el método de consenso deriva estimaciones cuantitativas a través de enfoques cualitativos. El Ministerio de Salud de España (1) y el Centro para el Control y Prevención de Enfermedades, CDC, (2) indican que el método de consenso, incluyendo el grupo nominal y las técnicas de Delphi, son útiles cuando es necesario un acuerdo de expertos sobre la formulación de recomendaciones, especialmente cuando la evidencia científica no está disponible, la evidencia existente esta disputa, o hay un bajo balance riesgo-beneficio. Helmer \& Rescher (3) discuten que el método de consenso representa "un nuevo enfoque epistemológico de las ciencias inexactas. El propósito de toda ciencia es explicar y predecir de una manera objetiva. Mientras que en las ciencias exactas la explicación y predicción tienen la misma estructura lógica, no es así en las ciencias inexactas. Esto permite diversas innovaciones metodológicas en las ciencias inexactas, por ejemplo, el juicio de expertos y la simulación" (p.25).

Generalmente, se utiliza un método de consenso para lograr un acuerdo en la toma de decisiones y responder a preguntas de investigación (Jones y Hunter, 1995). Como método de investigación, el resultado deseado es una solución, o un acuerdo general y convergencia de opiniones de expertos, en respuesta a una pregunta de investigación bien definida (4). El método de consenso también se utiliza en el desarrollo de programas y procesos de toma de decisiones de gestión para resolver problemas, generar nuevas ideas y determinar prioridades (5). El método de consenso se ha utilizado en todas las industrias, incluyendo los servicios de salud, ingeniería, gestión y psicología. En el sector salud, el método de consenso se ha utilizado en diversas disciplinas, como odontología (6), medicina (7), salud mental (8), obstetricia (9), enfermería (10), farmacia (11) y salud pública (12). En el cuadro 1 se presentan los elementos generales, con descripciones breves, para ambas técnicas.

Los investigadores que buscan responder preguntas de investigación a través de un riguroso proceso de consenso deben considerar la técnica de Delphi. El propósito de este artículo es proporcionar una visión general de la técnica Delphi como un importante método de consenso para avanzar la investigación en los servicios de salud en Latino América. El artículo describirá brevemente la historia del método de consenso, revisará la técnica Delphi, incluyendo los elementos del consenso, la composición del panel de expertos y las rondas de recolección de datos. Además, el artículo discute cómo superar las limitaciones básicas para maximizar la fiabilidad y la validez del proceso para obtener consenso.

\section{MÉTODO DE CONSENSO}

El método de consenso se originó a partir de una serie de estudios realizados por la RAND en la década de 1950 (4). El propósito de estos estudios fue desarrollar un proceso para que los militares obtengan el consenso más confiable de un grupo de expertos (13). La técnica Delphi fue desarrollada para someter a los expertos a "una serie de cuestionarios intercalados en profundidad de opinión controlada con retroalimentación" (14) (p.458). Aunque es una técnica que está madurando (15), Delphi es el método de consenso más común para investigadores de servicios de salud (16) para recopilar datos, sintetizar información y generar conocimiento a partir de confrontar la evidencia (17).

El otro método de consenso comúnmente utilizado, la técnica nominal del grupo, fue desarrollada por (18) para generar ideas principalmente. Sin embargo, la técnica se ha utilizado para lograr consenso en ambientes que no son de investigación, tales como paneles, grupos de trabajo y cultos. En general, la técnica de Delphi puede describirse como un enfoque multi-fase para desarrollar y confirmar el consenso de expertos a través de un proceso científico riguroso (19), mientras que la técnica de grupo nominal es un proceso flexible que pretende generar nuevas ideas como, así como lograr el acuerdo de los participantes (11). En el cuadro 2, se presenta un resumen de las principales características de la técnica de Delphi y del grupo nominal.

\section{Consenso definido}

Según Jones y Hunter (16), el enfoque del método de consenso "se encuentra donde la unanimidad de opiniones no existe debido a la falta de evidencia científica o donde hay evidencia contradictoria sobre un tema" (p.371). El método intenta evaluar el grado de acuerdo (medición de consenso) y resolver el desacuerdo (desarrollo de consenso). El consenso, según Avella (20), no significa un $100 \%$ de acuerdo, ya que podría ser difícil conseguir grupos de expertos que representen diferentes grupos con diferentes puntos de vista, perspectivas y prioridades para 
llegar a la unanimidad. Hutchings, Raine, Sanderson \& Black (21) describieron al consenso como un proceso grupal que involucraba una muestra "nominal" de unas 10 personas que incluye una reunión para discutir áreas de desacuerdo. La técnica Delphi alcanza un consenso entre el 51\% (22) y el 100\% de acuerdo (23), y con el 75\% se considera el umbral mínimo aceptable (24).

\begin{tabular}{|c|c|}
\hline Elementos & Breve descripción \\
\hline Participantes & $\begin{array}{l}\text { Se invita a los miembros a participar; los miembros individuales } \\
\text { son elegidos debido a sus competencias en contenidos específicos } \\
\text { y / o conocimiento del proceso; a menudo los miembros son } \\
\text { seleccionados debido a su experticia en la materia y la familiaridad } \\
\text { con la evidencia científica en este ámbito. }\end{array}$ \\
\hline Anonimato & $\begin{array}{l}\text { Realizado con cuestionarios o votación electrónica; proporciona a } \\
\text { los miembros la libertad de ofrecer opiniones evitando dominio, } \\
\text { eliminando la presión de los compañeros y eliminando las críticas } \\
\text { directas de otros miembros; evita la influencia del sesgo de "status" } \\
\text { de otros miembros. }\end{array}$ \\
\hline Iteración & $\begin{array}{l}\text { Los procesos se producen a través de múltiples rondas; permite a } \\
\text { los miembros cambiar de opinión; el número de rondas se fija con } \\
\text { anticipación o se determina según un criterio de consenso. }\end{array}$ \\
\hline $\begin{array}{l}\text { Retroalimentación } \\
\text { controlada }\end{array}$ & $\begin{array}{l}\text { La distribución de la respuesta grupal (indicando a cada individuo } \\
\text { sus propias respuestas en las rondas anteriores); la } \\
\text { retroalimentación se presenta en forma de un resumen estadístico } \\
\text { simple de la respuesta grupal; se pueden presentar contribuciones } \\
\text { individuales incluyendo argumentos, retroalimentación y } \\
\text { evidencia; todos los miembros están autorizados a aportar } \\
\text { información al proceso. La primera ronda suele ser un inventario; } \\
\text { la segunda ronda proporciona clasificaciones con contribuciones } \\
\text { individuales; las tercera y rondas adicionales permiten a los } \\
\text { miembros afinar las clasificaciones basadas en las contribuciones } \\
\text { del grupo. }\end{array}$ \\
\hline Grupo estadístico & $\begin{array}{l}\text { Las clasificaciones individuales y contribuciones de los miembros } \\
\text { se reconcilian en una respuesta grupal; la información es un } \\
\text { consenso, con clasificaciones, así como las opiniones de los } \\
\text { expertos. Sin embargo, las contribuciones disidentes son a menudo } \\
\text { registradas y reportadas. }\end{array}$ \\
\hline \multicolumn{2}{|c|}{$\begin{array}{l}\text { Adaptado de: van Teijlingen, Pitchforth, Bishop, and Russell, (19); Jones \& Hunter (16); } \\
\text { Woudenberg (41); Rowe et al. (47); Pill (34). }\end{array}$} \\
\hline
\end{tabular}

Cuadro 1. Elementos generales de las técnicas del método de consenso

Alcanzar el consenso de expertos puede parecer arbitrario a primera vista (25); sin embargo, el metodólogo está de acuerdo en que el enfoque a priori, o primero estipular el método de determinar el consenso, la definición de consenso y el proceso para reportar el consenso, incluyendo las perspectivas de la mayoría y minoría, es esencial para establecer la confiabilidad y validez de un estudio Delphi $(26,27)$. Incluso cuando el consenso se logra en condiciones ideales, investigación adicional y revisión de la evidencia recién publicada es necesaria para mantener continuamente la validez de las conclusiones de consenso (28).

\section{TÉCNICA DELPHI}

Dalkey (29) proporcionó la definición más temprana para la técnica de Delphi como "un conjunto de procedimientos para obtener $\mathrm{y}$ 
clarificar las opiniones de un grupo de personas. En la práctica, los procedimientos se utilizarían con un grupo de expertos o especialmente individuos bien informados" (p.1). Con más conocimientos, Linstone \& Turoff (30) ampliaron la definición a "un método para estructurar un proceso de comunicación grupal para que así el proceso sea eficaz en permitir que un grupo de individuos, como un todo, para hacer frente a un problema complejo " (p.3). El nombre tiene origen en el oráculo de Delfos, donde los antiguos griegos recibieron pronósticos de eventos futuros de Apolo (28). La técnica proporciona un enfoque grupal para la comunicación estructurada (30) para discutir y evaluar la efectividad de proyectos, procesos y / o prácticas complejas a través de una serie de cuestionarios (31). El resultado clave de la implementación de la técnica Delphi es el consenso, o el acuerdo grupal. El consenso, como concepto y elemento crítico de la técnica Delphi, se define en la siguiente sección.

\begin{tabular}{|l|l|}
\hline \multicolumn{1}{|c|}{$\begin{array}{c}\text { Técnica Delphi } \\
\text { Características / Pasos }\end{array}$} & \multicolumn{1}{|c|}{$\begin{array}{c}\text { Técnica Nominal Grupal } \\
\text { Características / Pasos }\end{array}$} \\
\hline $\begin{array}{l}\text { 1. Desarrollo de una pregunta de } \\
\text { investigación y el protocolo a priori con el } \\
\text { proceso Delphi. }\end{array}$ & $\begin{array}{l}\text { 1. Formulación y presentación de la } \\
\text { pregunta nominal. }\end{array}$ \\
\hline $\begin{array}{l}\text { 2. Selección de expertos para desarrollar el } \\
\text { panel. }\end{array}$ & 2. Generación silenciosa de ideas por escrito \\
\hline $\begin{array}{l}\text { 3. Utilice un cuestionario anónimo postal o } \\
\text { electrónico, con preguntas sí / no, } \\
\text { clasificación / calificación, y / o elementos } \\
\text { abiertos en múltiples rondas. }\end{array}$ & $\begin{array}{l}\text { 3. Retroalimentación de los miembros del } \\
\text { grupo para registrar cada idea en una frase } \\
\text { sucinta en un rotafolio. }\end{array}$ \\
\hline $\begin{array}{l}\text { 4. Proporcionar retroalimentación, mediante } \\
\text { el suministro de datos numéricos por sí solos } \\
\text { (por ejemplo, medios y frecuencias), con } \\
\text { comentarios específicos o resúmenes. }\end{array}$ & $\begin{array}{l}\text { 4. Discusión grupal de cada idea a su vez } \\
\text { para su aclaración y evaluación. }\end{array}$ \\
\hline $\begin{array}{l}\text { 5. Repetición de las rondas hasta que haya } \\
\text { una convergencia de opinión, por criterios } \\
\text { predeterminados sobre el consenso grupal, la } \\
\text { estabilidad de los juicios individuales, o el } \\
\text { número de rondas. }\end{array}$ & $\begin{array}{l}\text { 5. La votación individual sobre las ideas de } \\
\text { prioridad con la decisión grupal a menudo } \\
\text { se derivan matemáticamente a través de } \\
\text { clasificación o calificación. }\end{array}$ \\
\hline $\begin{array}{l}\text { 6. Resumir e informar los resultados del } \\
\text { consenso. }\end{array}$ & $\begin{array}{l}\text { 6. Retroalimentación de los resultados, } \\
\text { debate y nueva votación. }\end{array}$ \\
\hline Adaptado de: Cantrill et al. (11). \\
\hline
\end{tabular}

Cuadro 2. Características principales y pasos para los métodos de consenso

\section{INVESTIGACIÓN EN LOS SERVICIOS DE SALUD}

En el contexto de la investigación en los servicios de salud, la técnica de Delphi implica la retroalimentación grupal habitualmente recopilada por un cuestionario, donde los participantes revisan la evidencia científica y comparten la opinión de expertos, las perspectivas y las experiencias relacionadas con una pregunta de investigación específica (20). Usualmente, el cuestionario es un instrumento estructurado de recopilación de datos utilizado por los investigadores para organizar respuestas individuales a preguntas predefinidas. En la mayoría de los casos, los instrumentos tienen preguntas dicotómicas sí / no (32), así como preguntas abiertas para la retroalimentación cualitativa (33). Los elementos de respuesta numérica también pueden incluirse en los instrumentos para dar rango o clasificar algún contenido específico. El objetivo general es llegar a una conclusión válida, o consenso, de una deliberación grupal repetitiva (34) en respuesta a una pregunta de investigación bien definida (10). Keeney, Hasson \& McKenna (27) identificaron la técnica Delphi como un método esencial para lograr el consenso en temas donde ninguno existió 
previamente o la evidencia era limitada, de baja calidad y / o sujeto a debate.

Esta técnica requiere investigadores que seleccionen y comprometan a los participantes a compartir anónimamente sus comentarios con otros participantes en rondas iterativas para lograr el consenso. Un ejemplo de cómo la técnica Delphi se usa a menudo en la investigación de los servicios de salud es el desarrollo de una guía de práctica clínica siguiendo la clasificación de las Recomendaciones de Valoración, Desarrollo y Evaluación (GRADE). La técnica Delphi (35) guía a los investigadores en el trabajo con participantes expertos para sintetizar evidencias publicadas de calidad y disponibilidad variada, para compartir su experiencia y conocimiento como expertos y para considerar las preferencias del paciente para llegar a un consenso sobre cómo poder manejar mejor el proceso de enfermedad o un estado clínico $(36,37)$.

\section{Participantes}

Establecer un panel de expertos es un proceso útil en el que se pide a los profesionales conocedores del área de interés identificada que contribuyan en responder a una pregunta de investigación $(22,26)$. Además de la experiencia, los participantes deben estar interesados en el área de investigación, respetados dentro de su disciplina y dispuestos a participar en todo el proceso $(25,28)$. Aunque no existe una dimensión recomendada para el panel, los resultados pueden estar sesgados si no hay representación de las circunscripciones estudiadas. Los grupos heterogéneos, entre 10 y 20 participantes (30), de diversos orígenes tienden a producir resultados más confiables (38). Con el desarrollo de plataformas accesibles para la recolección de datos por Internet (39), los investigadores pueden incrementar la heterogeneidad del panel Delphi y la calidad de los participantes al involucrar a los principales expertos de todo el mundo en estos temas.

\section{Compromiso}

Mientras que la comunicación con los miembros del panel Delphi históricamente ocurrió a través de reuniones frente a frente y correo postal (34), en un primer estudio, Snyder-Halpern, Thompson, \& Schaffer (40) reportaron que las respuestas de correo electrónico, en comparación con las respuestas por correo, eran más legibles, facilitaron el ingreso de datos, mejoraron el rastreo de la comunicación, fueron menos costosos y mucho más rápidos. Aunque las tasas de respuesta pueden verse afectadas por cuestiones técnicas, incluidas las capacidades del sistema y las competencias tecnológicas de los participantes, éstas son menos difusas en la era digital actual. En la investigación contemporánea de Chang et al. (10) se encontró que la comunicación vía correo electrónico y encuesta en línea era fácil de usar para el usuario, facilitó respuestas individuales rápidas, y resultó en el compromiso de los participantes a lo largo de tres rondas. Recientemente, Toronto (39) informó que una plataforma de encuesta en línea incremento la participación en un estudio Delphi, ya que el proceso de recopilación de datos era más accesible, eficiente y oportuno. Sin embargo, para evitar errores críticos, los investigadores deben plantear cuidadosamente la pregunta de investigación, el diseño de instrumentos y el proceso específico para la recopilación de datos digitales.

\section{Anonimato}

Mantener el anonimato en un estudio Delphi permite a los participantes responder abiertamente $(11,34)$ y evita la influencia de personalidades dominantes (41) condicionando un falso consenso. Con la técnica Delphi, los investigadores deben permitir la libre expresión de perspectivas abiertas y honestas (25). El anonimato puro es a menudo difícil de lograr (16), ya que los participantes necesitan ser conocidos por el investigador para su retroalimentación y seguimiento $(25,26)$. Sin embargo, el anonimato completo de los investigadores y los participantes puede dar como resultado tasas de respuesta bajas y la falta de responsabilidad por las respuestas reflexivas $(25,28)$.

\section{Estructura de las Rondas}

La técnica de Delphi, que incorpora una serie de rondas iterativas, es un método para explorar la divergencia, así como el obtener consenso de un panel de expertos $(22,26)$. El número de rondas es un atributo importante para usar correctamente la técnica Delphi en la investigación. Sin embargo, no hay un número establecido de rondas $(25,42)$. Tan pocos como dos $(43)$ y tantos como diez (41) pueden ser necesarios para lograr el consenso. La mayoría de los investigadores indican que un estudio Delphi de tres rondas comúnmente alcanza un nivel aceptable de consenso $(10,22,42)$. El número de rondas se determina alcanzando un número especificado de rondas y / o al alcanzar el umbral de consenso 
establecido, definido previamente en el protocolo de investigación.

Debido al tiempo para completar cada ronda, más de tres rondas puede resultar en fatiga $\mathrm{y}$ desgaste de los participantes (25). Además, deben disponerse de recursos suficientes que incluyen tiempo y servicios administrativos para cada ronda (44). Según McMillan et al. (43), el tiempo mínimo para un Delphi de dos rondas es 30 días, aunque puede tardar más tiempo si los participantes requieren múltiples recordatorios para completar su trabajo. El tiempo requerido para cada ronda es importante debido a la disponibilidad de recursos (44) y porque la validez del consenso se ve afectada por las disminuciones en la varianza atribuida mayormente al desgaste (11). Por lo tanto, la tasa de atribución es un indicador importante para reportar los resultados. Con un estudio Delphi bien definido, el desgaste se reduce con el consenso grupal temprano alcanzado con tan sólo tres rondas (10).

\section{Progreso de las Rondas}

Delphi es una técnica múlti-etapas (34), y cada etapa construye en base la anterior (11). Según Woudenberg (41), la primera ronda es un inventario, o estimación, en el que se revisa una gran variedad de comentarios individuales, se agrupan y se devuelven a los participantes para en una segunda ronda más formativa. Cuando la primera ronda no está estructurada (28), se puede recoger una gran cantidad de datos mal definidos e información ambigua $(27,41)$. Sin embargo, McKenna (22) señaló que este problema se puede eliminar cuando la primera ronda se organiza y se estructura de manera "reactiva". Esto significa que los participantes reciben información de antecedentes, incluyendo información científica, oportunidades definidas para retroalimentación e instrumentos para forzar el puntaje y para guiar respuestas abiertas.

En general, la primera ronda reactiva (22) facilita una progresión organizada de un tema amplio a un área más específica relacionada con la pregunta de investigación en rondas posteriores (10). Este tipo de primera ronda se recomienda para los investigadores que buscan completar un estudio Delphi de manera clara y concisa, con tiempo limitado y de una forma rentable. Aunque existe un riesgo de sesgo debido a respuestas limitadas con una primera ronda reactiva (22), esto puede ser mitigado al estimular la retroalimentación y la discusión a través de cada ronda (10). El estímulo propicio al proporcionar respuestas anónimas individuales a todo el panel estimula a los participantes a expresar continuamente sus puntos de vista (27).

Después de la primera ronda, se analizan los datos recopilados durante cada ronda subsiguiente, incluidos los elementos del instrumento, los comentarios abiertos y los rangos / clasificaciones, entre y a lo largo de las rondas (27). Los investigadores deben recolectar todos los datos, organizar las respuestas individuales, resumir los resultados y devolver los datos como información al panel (11), generalmente con las respuestas individuales y los totales de cada ronda. Los investigadores también son responsables de responder a las preguntas de los participantes y aclarar áreas de confusión cuando sea necesario. Chang et al. (10)reporto, que "este método de retroalimentación controlada facilitó el desarrollo de investigaciones previas y dio a los participantes la oportunidad de cambiar sus opiniones después de revisar las respuestas anónimas de otros miembros del panel" (p. 2329).

\section{CONCLUSIÓN}

La técnica Delphi es un poderoso método de investigación cualitativa que requiere la formación de un panel interactivo de expertos. La técnica Delphi es un proceso participativo que involucra interacciones entre el investigador y los participantes con experiencia relacionada a la pregunta de investigación; esta interacción generalmente ocurre a través de una serie de cuestionarios electrónicos completados por los participantes o por correo electrónico (31). Para que el proceso sea efectivo, los participantes deben estar dispuestos a compartir sus conocimientos y trabajar hacia la solución consensuada en cuestiones de experiencia y opinión. Linstone y Turoff (30) sugieren algunos criterios para situaciones en las que se debe considerar la técnica Delphi: 1) El problema de investigación no se presta a técnicas analíticas precisas, pero puede beneficiarse de juicios subjetivos en base colectiva; 2) La población de investigación presenta diversos antecedentes con respecto a la experiencia y experticia; 3) se requieren más sujetos que los que pueden interactuar efectivamente frente a frente; y 4) El tiempo, los costos y la logística hacen que las reuniones frecuentes de todos los sujetos sean inviables.

Aunque la técnica Delphi es un proceso establecido para lograr consenso a través de un proceso sistemático de recolección de información para responder a una pregunta de investigación (33), la forma en que el consenso se 
define y operacionaliza puede variar de estudio a estudio dependiendo de los objetivos de investigación (38). Para los investigadores, el aspecto más difícil de llevar a cabo un buen estudio Delphi es la formulación de preguntas, la prevención de sesgos durante la interacción con los participantes y la codificación y clasificación de los datos para la retroalimentación a los participantes (45). A pesar de estos desafíos, Fink, Kosecoff, Chassin \& Brook (46) argumentan que la técnica Delphi crea un "entorno estructurado en el que se da a los expertos la mejor información disponible, permitiendo que sus soluciones a los problemas sean más justificables y creíbles que entonces" (p. 979).

\section{REFERENCIAS BIBLIOGRÁFICAS}

1. GuíaSalud. Elaboración de guías de práctica clínica en el sistema nacional de salud: Actualización del manual metodológico. Madrid, Espana: Ministerio de Sanidad, Servicios Sociales e Igualdad; 2016. Available from: http://portal.guiasalud.es/emanuales/elabora cion_2/Capitulos/completo.pdf.

2. Centers for Disease Control and Prevention. Gaining consensus among stakeholders through the nominal group technique. Evaluation Briefs. 2006;7:1-3.

3. Helmer O, Rescher N. On the epistemology of the inexact sciences. Manag Sci. 1959;6(1):25-52.

https://doi.org/10.1287/mnsc.6.1.25

4. Okoli C, Pawlowski SD. The Delphi method as a research tool: an example, design considerations and applications. Information \& Management. 2004;42(1):15-29. https://doi.org/10.1016/j.im.2003.11.002

5. Delbecq AL, Van de Ven A, Gustafson DH. Group techniques for program planning: a guide to nominal group and Delphi processes. Middleton, WI: Green Briar Press; 1996.

6. Cramer CK, Klasser GD, Epstein JB, Sheps SB. The Delphi process in dental research. J Evid Based Dent Pract. 2008;8(4):211-20. https://doi.org/https://doi.org/10.1016/j.jebd p.2008.09.002

7. Gallagher M, Hares TIM, Spencer J, Bradshaw C, Webb IAN. The nominal group technique: A research tool for general practice? Fam Pract. 1993;10(1):76-81. https://doi.org/10.1093/fampra/10.1.76

8. Hardy DJ, O'Brien AP, Gaskin CJ, O'Brien AJ, Morrison-Ngatai E, Skews G, et al.
Practical application of the Delphi technique in a bicultural mental health nursing study in New Zealand. J Adv Nurs. 2004;46(1):95109. https://doi.org/doi:10.1111/j.13652648.2003.02969.x

9. Kennedy HP. A model of exemplary midwifery practice: results of a Delphi study. J Midwifery Women Health. 2000;45(1):4$19 . \quad$ https://doi.org/10.1016/S15269523(99)00018-5

10. Chang AM, Gardner GE, Duffield C, Ramis M-A. A Delphi study to validate an advanced practice nursing tool. J Adv Nurs. 2010;66(10):2320-30.

https://doi.org/10.1111/j.13652648.2010.05367.x

11. Cantrill JA, Sibbald B, Buetow S. The Delphi and nominal group techniques in health services research. Int J Pharm Pract. 1996;4(2):67-74. https://doi.org/doi:10.1111/j.20427174.1996.tb00844.x

12. Ferri CP, Prince M, Brayne C, Brodaty H, Fratiglioni L, Ganguli M, et al. Global prevalence of dementia: a Delphi consensus study. Lancet. 2005;366(9503):2112-7. https://doi.org/10.1016/S01406736(05)67889-0

13. Rowe G, Wright G. The Delphi technique: past, present, and future prospects Introduction to the special issue. Technol Forecast Soc Change. 2011;78(9):1487-90. https://doi.org/10.1016/j.techfore.2011.09.0 02

14. Dalkey N, Helmer O. An experimental application of the DELPHI method to the use of experts. Manag Sci. 1963;9(3):458-67. https://doi.org/10.1287/mnsc.9.3.458

15. Kennedy HP. Enhancing Delphi research: methods and results. J Adv Nurs. 2004;45(5):504-11.

https://doi.org/10.1046/j.13652648.2003.02933.x

16. Jones J, Hunter D. Qualitative research: consensus methods for medical and health services research. BMJ. 1995;311(7001):376-80. https://doi.org/10.1136/bmj.311.7001.376

17. Roberts-Davis M, Read S. Clinical role clarification: using the Delphi method to establish similarities and differences between nurse practitioners and clinical nurse specialists. J Clin Nurs. 2001;10(1):3343. $\quad \mathrm{https} / / /$ doi.org/10.1046/j.13652702.2001.00437.x

18. Van de Ven AH, Delbecq AL. The nominal group as a research instrument for 
exploratory health studies. Health Am J Public Health. 1972;62(3):337-42. https://doi.org/10.2105/ajph.62.3.337

19. van Teijlingen E, Pitchforth E, Bishop C, Russell E. Delphi method and nominal group technique in family planning and reproductive health research. J Fam Plann Reprod Health Care. 2006;32(4):249-52. https://doi.org/10.1783/1471189067785865 98

20. Avella JR. Delphi panels: research design, procedures, advantages, and challenges. International Journal of Doctoral Studies. 2016;11:305-21. https://doi.org/10.28945/3561

21. Hutchings A, Raine R, Sanderson C, Black $\mathrm{N}$. A comparison of formal consensus methods used for developing clinical guidelines. J Health Serv Res Policy. 2006;11(4):218-24. https://doi.org/10.1258/1355819067784765 53

22. McKenna HP. The Delphi technique: a worthwhile research approach for nursing? J Adv Nurs. 1994;19(6):1221-5. https://doi.org/10.1111/j.1365-

2648.1994.tb01207.x

23. Williams PL, Webb C. The Delphi technique: a methodological discussion. J Adv Nurs. 1994;19(1):180-6. https://doi.org/10.1111/j.1365-

2648.1994.tb01066.x

24. Diamond IR, Grant RC, Feldman BM, Pencharz PB, Ling SC, Moore AM, et al. Defining consensus: a systematic review recommends methodologic criteria for reporting of Delphi studies. J Clin Epidemiol. 2014;67(4):401-9. https://doi.org/10.1016/j.jclinepi.2013.12.00 2

25. Keeney S, Hasson F, McKenna HP. A critical review of the Delphi technique as a research methodology for nursing. Int J Nurs Stud. https://doi.org/10.1016/S00207489(00)00044-4

26. Hasson F, Keeney S, McKenna H. Research guidelines for the Delphi survey technique. J Adv Nurs. 2000;32(4):1008-15. https://doi.org/doi:10.1046/j.13652648.2000.t01-1-01567.x

27. Keeney S, Hasson F, McKenna H. Consulting the oracle: ten lessons from using the Delphi technique in nursing research. J Adv Nurs. 2006;53(2):205-12. https://doi.org/10.1111/j.1365-

2648.2006.03716.x
28. Powell C. The Delphi technique: myths and realities. Journal of Advanced Nursing. 2003;41(4):376-82.

https://doi.org/10.1046/j.13652648.2003.02537.x

29. Dalkey NC. Delphi. Santa Clara, CA: The Rand Corporation; 1967.

30. Linstone HA, Turoff M, editors. The delphi method: techniques and applications. Boston, MA: Addison-Wesley; 1975.

31. Yousuf MI. Using experts' opinions through Delphi technique. Practical Assessment, Research, \& Evaluation. 2007;12(4):1-8.

32. Kalaian SA, Kasim RM. Terminating sequential Delphi survey data collection. Practical Assessment, Research \& Evaluation. 2012;17(5):1-10.

33. Hsu C-C, Sandford BA. The Delphi technique: making sense of consensus. Practical Assessment, Research, \& Evaluation. 2007;12(10):1-8.

34. Pill J. The Delphi method: substance, context, a critique and an annotated bibliography. Socio-Economic Planning Sciences. 1971;5(1):57-71. https://doi.org/10.1016/00380121(71)90041-3

35. Jaeschke R, Guyatt $G H$, Dellinger $P$, Schünemann H, Levy MM, Kunz R, et al. Use of GRADE grid to reach decisions on clinical practice guidelines when consensus is elusive. BMJ. 2008;337:a744. https://doi.org/10.1136/bmj.a744

36. Guyatt GH, Oxman AD, Kunz R, FalckYtter Y, Vist GE, Liberati A, et al. Going from evidence to recommendations. BMJ. 2008;336(7652):1049-51. https://doi.org/10.1136/bmj.39493.646875. $\mathrm{AE}$

37. Guyatt GH, Oxman AD, Vist GE, Kunz R, Falck-Ytter $\mathrm{Y}$, Alonso-Coello $\mathrm{P}$, et al. GRADE: an emerging consensus on rating quality of evidence and strength of recommendations. BMJ. 2008;336(7650):924-6. https://doi.org/10.1136/bmj.39489.470347. $\mathrm{AD}$

38. Black N, Murphy M, Lamping D, McKee M, Sanderson C, Askham J, et al. Consensus development methods: a review of best practice in creating clinical guidelines. $\mathrm{J}$ Health Serv Res Policy. 1999;4(4):236-48. https://doi.org/10.1177/1355819699004004 10

39. Toronto C. Considerations when conducting e-Delphi research: a case study. Nurse Res. 
2017;25(1):10-5.

https://doi.org/10.7748/nr.2017.e1498

40. Snyder-Halpern R, Thompson CB, Schaffer J, editors. Comparison of mailed vs. Internet applications of the Delphi technique in clinical informatics research. Proceedings of the AMIA Symposium; 2000; Los Angeles, CA: American Medical Informatics Association.

41. Woudenberg F. An evaluation of Delphi. Technol Forecast Soc Change. 1991;40(2):131-50.

https://doi.org/10.1016/00401625(91)90002-W

42. Pulcini J, Wilbur J, Allan J, Hanson C, Uphold CR. Determining criteria for excellence in nurse practitioner education: use of the Delphi Technique. Nurs Outlook. 2006;54(2):102-10.

https://doi.org/10.1016/j.outlook.2005.09.01 0

43. McMillan SS, King M, Tully MP. How to use the nominal group and Delphi techniques. Int J Clin Pharm. 2016;38(3):655-62.

https://doi.org/10.1007/s11096-016-0257-x

44. Duffield C. The Delphi technique: a comparison of results obtained using two expert panels. Int $\mathrm{J}$ Nurs Stud. 1993;30(3):227-37.

https://doi.org/10.1016/0020-

7489(93)90033-Q

45. Davidson PL. The Delphi technique in doctoral research: considerations and rationale. Review of Higher Education and Self-Learning. 2013;6(22):53-65.

46. Fink A, Kosecoff J, Chassin M, Brook RH. Consensus methods: characteristics and guidelines for use. Am J Public Health. 1984;74(9):979-83. https://doi.org/10.2105/ajph.74.9.979

47. Rowe G, Wright G, Bolger F. Delphi: a reevaluation of research and theory. Technol Forecast Soc Change. 1991;39(3):235-51. https://doi.org/10.1016/00401625(91)90039-I 